\title{
Formación de estudiantes universitarios en responsabilidad social: Hacia la construcción de una propuesta metodológica
}

\author{
Training university students in social responsibility: Towards the construction of a \\ methodological proposal
}

\author{
ROJO-CARLÓN, José María†, RIVERA-IRIBARREN, Maricel y CALDERÓN-SOTO, Lorena
}

Instituto Tecnológico de Sonora

ID $1^{\text {er }}$ Autor: José María, Rojo-Carlón / ORC ID: 0000-0003-3502-3379, arXiv Author ID: 3467884, CVU CONACYT ID: 1074691

ID $1^{\text {er }}$ Coautor: Maricel, Rivera-Iribarren / ORC ID: 0000-0003-1823-0149, Researcher ID Thomson: S-7893-2018, CVU CONACYT ID: 896629

ID $2^{\text {do }}$ Coautor: Lorena, Calderón-Soto / ORC ID: 0000-0002-8407-831X, Researcher ID Thomson: S-7886-2018, CVU CONACYT ID: 22017

DOI: $10.35429 /$ JET.2020.11.4.13.22

Recibido: 05 de Marzo, 2020; Aceptado 20 de Junio, 2020

\section{Resumen}

La Responsabilidad Social Universitaria, es un fenómeno complejo en donde convergen diferentes procesos que se desarrollan en las máximas casas de estudio, quienes para cumplir con la misión que les ha sido encomendada, deben orientar sus funciones hacia la transformación social. Si bien, el concepto ha evolucionado permitiendo a las Instituciones de Educación Superior su adaptabilidad al contexto y a sus necesidades, aún resulta necesario contar con un marco de referencia común que guíe su actuar, específicamente en materia de responsabilidad social (RS), que les permita formar profesionales responsables no solo con sus acciones, sino de los impactos que generan a nivel social y ambiental. Este estudio de corte cualitativo - fenomenológico, tiene por objetivo identificar las orientaciones metodológicas para la formación en RS con base en las experiencias recopiladas de proyectos con enfoque socialmente responsable en 13 universidades mexicanas y latinoamericanas, utilizando una muestra de tipo teórica. Los principales hallazgos plantean seis categorías emergentes para la formación en RS: a) empatía comunitaria; b) participación ciudadana; c) vinculación con organizaciones; d) acompañamiento docente; e) diálogo social y; f) aplicación del conocimiento, a partir de las cuales se presenta una propuesta metodológica para su implementación.

Responsabilidad social Universitaria, Formación en responsabilidad social, Orientaciones metodológicas

\begin{abstract}
University Social Responsibility is a complex phenomenon where different processes that are developed in the Higher Education Institutions converge. The universities must accomplish the mission that was entrusted to them and they must guide their functions to social transformation. Although the concept has evolved allowing Higher Education Institutions to adapt it to the context and their needs, it is still necessary to have a common framework to guide their actions, specifically regarding the social responsibility, that allow them to train responsible professionals, not only with their actions, but also for the impacts generated in a social and environmental level. This study uses a qualitative approach using a phenomenological method and its aim is to identify the methodological orientations to train students in Social Responsibility based on the experiences collected from socially responsible projects in 13 mexican and latin american universities. The main results present six emergent categories to train in SR: a) communitary empathy, b) citizen participation, c) linking with organizations, d) teaching accompaniment, e) social dialog and f) knowledge application, from which a methodological proposal is presented.
\end{abstract}

University Social Responsibility, Socially responsible training, Methodological orientations

Citación: ROJO-CARLÓN, José María, RIVERA-IRIBARREN, Maricel y CALDERÓN-SOTO, Lorena. Formación de estudiantes universitarios en responsabilidad social: Hacia la construcción de una propuesta metodológica . Revista Teoría Educativa. 2020. 4-11: 13-22

$\dagger$ Investigador contribuyendo como primer autor. 


\section{Introducción}

La Responsabilidad Social Universitaria (RSU) es un fenómeno complejo que comprende distintos procesos de las Instituciones de Educación Superior (IES). La gestión interna de la universidad, la formación estudiantil mediante la docencia y la investigación producida por los miembros de los Cuerpos Académicos son los pilares que Vallaeys (2014) delimita para la RSU, los cuales deberán estar encaminados a resolver las problemáticas del contexto de la sociedad actual, de tal manera que la universidad debe perfilarse como una directriz para la transformación social (Pérez, 2016).

Las IES son las encargadas de la formación de profesionistas y ciudadanos responsables (Ayala et. al., 2019). Si las universidades no se comprometen a formar en esta última característica, su futuro como transformadora de contextos puede verse comprometido al no lograrse los objetivos de desarrollo sustentable y alternativas de mejora para la comunidad en la que se asientan.

A pesar de que la transformación positiva del entorno es uno de los fines de la RSU, no todas las universidades orientan sus acciones para alcanzarlo (Beltrán, et. al., 2014). Ojeda y Álvarez (2015), plantean que las universidades se preocupan por formar seres humanos competentes para la vida laboral, pero si no existe un enfoque de sensibilización ante situaciones del contexto actual, difícilmente podrán contribuir a solventar las necesidades reales dadas por el mismo.

Según Gaete (2015), es necesario el involucramiento de los estudiantes con el entorno durante su formación, ya que esto los llevará a un proceso de concientización ciudadana, que se logrará a través de la práctica en un ámbito real por medio de la investigación, docencia y las políticas internas de gestión, ya que su participación en proyectos académicos y/o voluntariado contribuirá a mejorar la percepción que ellos tienen de los problemas sociales e impactará en su actuar ante los mismos.

De acuerdo con Ojeda y Álvarez (2015), el prestigio de las universidades podría verse afectado al no contar con tres aspectos fundamentales:
1. Políticas, lineamientos y normas para el cuidado de su comunidad.

2. Líderes con sentido de comunidad que permitan responder a las necesidades sociales y/o medioambientales.

3. Modelos de gestión que respeten y soporten el desarrollo sustentable.

Además Calles et. al. (2018), destacan lo fundamental que es para las organizaciones el difundir y socializar el trabajo que se realiza en materia de RSU, ya que estas acciones generan una percepción positiva en la comunidad universitaria, debido a que pueden conocer todo el esfuerzo y las acciones que la administración realiza en este tenor. En este sentido Alférez (2014), afirma que si las Instituciones de Educación Superior no fomentan la responsabilidad desde sus procesos de gestión, las actividades tanto docentes como de investigación no tendrán un verdadero impacto en el contexto que la rodea y no contribuirán de manera efectiva en el desarrollo de las sociedades. Aunado a ello, el no hacerlo puede afectar la reflexión del estudiantado sobre su propia práctica, así como en la formación de actitudes y valores relacionadas con las problemáticas de la sociedad, y en general su educación integral.

Ahumada, et. al. (2018), afirman que al no incluir la responsabilidad social desde el currículum macro, no se podrá ver reflejada dentro de las actividades áulicas, en el desarrollo integral de los futuros profesionistas e incluso al momento de ser empleados por alguna organización; reafirmando que la formación que se recibe en las IES se basa primordialmente en el desarrollo de competencias laborales.

Este déficit, coloca en las puertas de las universidades un área de oportunidad en el desarrollo de un sistema de formación en responsabilidad social que permita formar profesionales responsables, no solo con sus acciones, sino de los impactos que generan a nivel social y ambiental. En este sentido, el objetivo de esta investigación es determinar las orientaciones metodológicas para la formación en responsabilidad social con base en las experiencias de proyectos con enfoque socialmente responsable en universidades de México y Latinoamérica. 
A principios del siglo XXI, algunas de las máximas casas de estudio latinoamericanas comenzaron a establecer sus propias orientaciones sobre cómo las formadoras de profesionistas deberían vivir la responsabilidad social; dando inicio con alianzas como el Observatorio Regional de Responsabilidad Social para América Latina y el Caribe (ORSALC), el Observatorio Mexicano de Responsabilidad Social Universitaria (OMERSU) y la Unión de Responsabilidad Social Universitaria de Latinoamérica (URSULA) (Vallaeys, 2018).

Uno de los puntos que las universidades tomaron en cuenta para la adaptación de la Responsabilidad Social al contexto de la formación fue el de los impactos, dividiéndolos en dos ejes: (1) organizacional, en la cual sus efectos internos y externos se ven relacionados a aspectos laborales y ambientales; y (2) el eje académico, cuyos resultados pueden observarse en el ámbito educativo, relativo a la formación estudiantil, y el cognitivo, relacionado a la investigación y generación de conocimientos (Vallaeys, 2014). Lo cual en palabras de Schwalb, et. al. (2019), permite establecer cuatro ámbitos de gestión socialmente responsable: la formación, cognición, participación social y la gestión organizacional.

La formación consiste en la educación de "profesionales ciudadanos, la gestión responsable de la planificación curricular, los contenidos de las asignaturas, la metodología pedagógica y la propuesta didáctica". Es decir, la formación se refiere a todo lo relacionado con el diseño e implementación del currículum, mismo que deberá estar apegado a la resolución de problemáticas que aquejan en la actualidad y será consultado con agentes a los que pueda impactar; mientras que el proceso de enseñanzaaprendizaje deberá ser planteado con base en proyectos de colaboración con entidades sociales (Schwalb, et. al., 2019).

En segundo lugar, se presenta el ámbito de gestión organizacional, cuyas acciones van orientadas al cumplimento de la legislación por la que se rige la universidad, tanto interna como externa, el respeto a los derechos de las y los trabajadores, al trato digno de los mismos, rendición de cuentas, desarrollo sustentable y la promoción de la RSU en la institución (Barroso, Santos y Patrón, 2016).
El tercer ámbito corresponde a la cognición, cuyo papel se encarga de "la investigación, la producción y la difusión del saber" (Schwalb, et. al., 2019). Lo anterior hace referencia al deber de la universidad de alinear los conocimientos para que estos contribuyan al desarrollo de nuevas tecnologías y esquemas sociales para impactar de manera positiva y transdisciplinar en la solución de problemas. Finalmente, el cuarto elemento, compuesto por la participación social, concierne al vínculo creado entre los actores internos de la universidad, llámese alumnos, personal académico y administrativo, y los agentes externos, comprendidos como organizaciones sociales, empleadores, otras universidades, colectivos locales y demás instancias; recayendo sobre ellos el interés de saber cómo impactará la formación del estudiantado como profesionales $y$ agentes sociales de cambio en el contexto (Vallaeys y Álvarez, 2019).

Durante la formación universitaria las y los estudiantes modifican sus habilidades y actitudes tanto a nivel personal como vocacional, es durante este período que su visión del mundo se amplía, comienza a vislumbrar las implicaciones de la profesión que ha seleccionado y para la cual se ha empezado a preparar; siendo este el momento preciso cuando se debe aprovechar para diversificar sus experiencias, presentarle desde un enfoque global las distintas problemáticas sociales en las que comunidad se encuentra inmersa, tarea que no puede dejarse en manos de un solo sector como usualmente se le ha conferido al empresarial.

Es así que, la responsabilidad de mostrarles el panorama futuro es menester de la universidad, la cual debe generar una visión integradora que le permita considerar no solo el desarrollo económico, sino también el social y el ambiental; siendo esta una ardua labor para el docente, teniendo que cumplir con el contenido de los programas a la vez que desarrolla habilidades y actitudes.

Sin embargo al plantearse la responsabilidad social como un eje de acción desde el aula, se le brinda la dirección que sus esfuerzos deben de tener, y es ahí donde en palabras de Guzman, et. al, (2015) "el docente deja de ser un consumidor del curriculum y se vuelve un actor, con una participación activa”. 
La responsabilidad social es un fenómeno complejo, a tal grado que para obtener resultados a nivel formación se requiere del involucramiento de todos sus actores; no basta abordarlo como un tema de discusión, el compromiso social debe verse reflejado tanto en los procesos administrativos como en el aula. Las universidades aún no están preparadas para verlo como un proceso integral o integrado, que se permee a través de cada acción del docente, por lo que es necesario establecer orientaciones metodológicas que sirvan de soporte, permitiéndole integrar este enfoque a los conocimientos disciplinares, la RS no tiene que ser un tema esporádico o especial, la RS tiene que ser parte del fundamento de la práctica docente.

\section{Método}

Para la selección de la muestra en la investigación cualitativa, esta tuvo que cumplir con tres elementos (Hernández, et. al., 2014): (1) la capacidad operativa de recolección y análisis, referida a que la cantidad de casos estudiados no es relevante, siempre y cuando se puedan analizar con calidad; (2) el entendimiento del fenómeno, es decir, que mediante la información recabada se pueda encontrar la intersubjetividad en las unidades de análisis permitiendo la saturación de las categorías emergentes; y (3) la naturaleza del fenómeno de análisis, es decir, si las características propias del constructo que se pretende estudiar permitirá el análisis agotando los recursos y el tiempo establecido en la investigación.

Se utilizó el tipo de muestra teórica, recopilando la información de manera documental, a través de los casos encontrados en el catálogo de Experiencias Exitosas de RSU en América Latina, publicado por URSULA y en el documento Prácticas y Modelos de Responsabilidad Social Universitaria en México de la Asociación Nacional de Universidades e Instituciones de Educación Superior (ANUIES, 2016).

Para el análisis de los datos se utilizó la propuesta de sistematización y análisis de Manig (2019):

1. Control y códigos: se establece cuál es el significado que se le dará a los signos que permiten identificar los informantes y unidad de análisis.
2. Control de informantes: se designa una letra distintiva para el control de cada fuente de información;

3. Codificación de los datos: se buscan las experiencias en cada uno de los casos por medio de la lectura.

4. Intersubjetividad: donde se pretende encontrar las vivencias con elementos similares; y finalmente el paso 7) Categorías emergentes: se agrupan las unidades de análisis y se les otorga un nombre con base en lo que las hace parecidas.

\section{Resultados y discusión}

En el presente trabajo de investigación se realizó el análisis de 13 casos de universidades nacionales e internacionales (dos de Argentina, cuatro de Colombia y siete de distintos Estados de la República Mexicana) relacionados directamente a la formación de las y los estudiantes en responsabilidad social.

\section{Categorías emergentes}

Las categorías emergentes fueron las siguientes: a) empatía comunitaria; b) participación ciudadana; c) vinculación con organizaciones; d) acompañamiento docente; e) diálogo social y f) aplicación del conocimiento, las cuales se describen a continuación.

\section{a) Empatía comunitaria}

Según las experiencias de éxito en RSU analizadas, es necesario que el estudiantado genere un proceso de concientización ante las situaciones que aquejan a las personas en el contexto actual antes de poder actuar en pro de su resolución. La empatía comunitaria es el paso previo al diseño de estrategias de solución; este momento consiste en la recolección de datos o el establecimiento de relaciones y lazos con la comunidad, el cual puede ser de manera indirecta o directa, mediante el análisis de la teoría o la introducción al campo, respectivamente. De igual manera, el objetivo máximo de la formación en responsabilidad social debe ser que el alumnado consiga incrementar la empatía sobre las situaciones que aquejan a una comunidad, esta misma deberá ser reflejada en cambios en sus comportamientos, donde demuestren que comprenden la realidad que los rodea y que se interesen por continuar su labor como agentes de transformación social. (Ver tabla1)

ROJO-CARLÓN, José María, RIVERA-IRIBARREN, Maricel y CALDERÓN-SOTO, Lorena. Formación de estudiantes universitarios en responsabilidad social: Hacia la construcción de una propuesta metodológica . Revista Teoría Educativa. 2020 


\begin{tabular}{|c|c|}
\hline Unidad de Análisis & Caso/Código \\
\hline $\begin{array}{l}\text { "...vivenciar las diferentes problemáticas } \\
\text { sociales comunitarias y desde allí comprender y } \\
\text { transformar el contexto social" }\end{array}$ & $\mathrm{C} 1 / \mathrm{E} 1$ \\
\hline $\begin{array}{l}\text { "realización de una lectura compleja y crítica de } \\
\text { un contexto determinado, promoviendo la } \\
\text { construcción de alternativas de acción" }\end{array}$ & C3/E3 \\
\hline $\begin{array}{l}\text { "Genera un sentimiento de comunidad el } \\
\text { entender que se está trabajando para el } \\
\text { mejoramiento de la calidad de vida de los/as } \\
\text { vecinos/a s" }\end{array}$ & C6E6 \\
\hline $\begin{array}{l}\text { "recogen testimonios que hablan de una visión } \\
\text { diferente de la realidad; aprendizaje en valores; } \\
\text { mayor conciencia social; concientización sobre } \\
\text { una problemática" }\end{array}$ & C1E3 \\
\hline $\begin{array}{l}\text { "Formación y capacitación, a través de distintos } \\
\text { eventos, sobre la temática" }\end{array}$ & C2E5 \\
\hline $\begin{array}{l}\text { "la inclusión curricular de los ODS, a partir de } \\
\text { cátedras transversales sobre Ética y } \\
\text { Sostenibilidad donde se aborda material } \\
\text { bibliográfico obligatorio sobre inclusión" }\end{array}$ & C2E7 \\
\hline $\begin{array}{l}\text { "se ha transformado mi manera de apreciar y } \\
\text { abordar el mundo de las personas con } \\
\text { capacidades diferentes, permitiéndole tener una } \\
\text { mente más abierta y consciente de lo colectivo" }\end{array}$ & C9E9 \\
\hline $\begin{array}{l}\text { "me dan inspiración para seguir con el trabajo } \\
\text { humanista y apasionante que se encarna en el } \\
\text { bibliotecario" }\end{array}$ & C9E10 \\
\hline
\end{tabular}

Tabla 1 Empatía comunitaria

\section{b) Participación ciudadana}

Esta categoría hace referencia a la intervención por parte de estudiantes dentro de las comunidades con el fin de aportar a la solución de problemas que se presentan en el entorno y va de la mano con la empatía comunitaria, debido a que esta motivará el desarrollo de estrategias e intervenciones en el campo de acción. Además, estas acciones de participación comunitaria tienen como finalidad aportar bagaje empírico a las primeras nociones de empatía generadas en el proceso de concientización y diseño de los proyectos de responsabilidad social. (Ver tabla 2)

\begin{tabular}{|c|c|}
\hline Unidad de Análisis & Caso/Código \\
\hline $\begin{array}{l}\text { "participando activamente en una organización } \\
\text { de la sociedad civil para poder abordar valores } \\
\text { que hacen al compromiso ciudadano, conciencia } \\
\text { ética, responsabilidad social" }\end{array}$ & C1E2 \\
\hline $\begin{array}{l}\text { "involucramiento progresivo y sostenido por } \\
\text { parte de directivos personal de administración y } \\
\text { servicio, docentes y alumnos" }\end{array}$ & C2E14 \\
\hline $\begin{array}{l}\text { "Práctica en Responsabilidad Social se } \\
\text { desarrolla en escenarios sociales y comunitarios } \\
\text { en articulación con organizaciones" }\end{array}$ & C3E8 \\
\hline $\begin{array}{l}\text { "participando activamente en una organización } \\
\text { de la sociedad civil para poder abordar valores } \\
\text { que hacen al compromiso ciudadano, conciencia } \\
\text { ética, responsabilidad social" }\end{array}$ & C1E2 \\
\hline $\begin{array}{l}\text { "reconociendo por medio de la participación } \\
\text { comunitaria las posibles actuaciones en pro de la } \\
\text { transformación social y la construcción de la } \\
\text { paz" }\end{array}$ & C4E14 \\
\hline
\end{tabular}

Tabla 2 Participación ciudadana

\section{c) Vinculación con organizaciones}

Según las experiencias analizadas, la formación en RSU necesita generarse en el ámbito del trabajo comunitario. Es por esto, que los proyectos deben estar vinculados a organizaciones que busquen solucionar los problemas detectados para el mejoramiento de los resultados. Además, el trabajo con organizaciones propicia la expansión del impacto de los resultados obtenidos por parte de las intervenciones y permite la participación de otros actores clave dentro de la comunidad. (Ver tabla 3)

\begin{tabular}{|c|c|}
\hline Unidad de Análisis & Caso/Código \\
\hline $\begin{array}{l}\text { "El estudiante se inserta en una } \\
\text { organización de la sociedad civil" }\end{array}$ & C1E6 \\
\hline $\begin{array}{lrr}\text { "estudiantes } & \text { se instalan en distintos } \\
\text { contextos } & \text { organizacionales } & \text { y } \\
\text { territoriales" } & & \\
\end{array}$ & C5E2 \\
\hline $\begin{array}{l}\text { "Vinculación de los docentes al contexto } \\
\text { desde las funciones de docencia e } \\
\text { investigación" }\end{array}$ & C5E6 \\
\hline $\begin{array}{l}\text { "vinculan diferentes actores de los } \\
\text { territorios" }\end{array}$ & C5E10 \\
\hline $\begin{array}{l}\text { "se han podido establecer lazos de } \\
\text { cooperación con algunas de las } \\
\text { comunidades más necesitadas de la } \\
\text { entidad oaxaqueña, permitiendo } \\
\text { fortalecer el desarrollo social y la } \\
\text { armonía de las comunidades" }\end{array}$ & C7E12 \\
\hline $\begin{array}{l}\text { "Se han atendido solicitudes y } \\
\text { colaborado de manera directa en diversas } \\
\text { entidades federativas" }\end{array}$ & C9E2 \\
\hline $\begin{array}{l}\text { "la Facultad de Agrobiología firmó un } \\
\text { convenio de colaboración con el ejido } \\
\text { Huatzirán, con el objetivo de que } \\
\text { profesores y alumnos cuenten con } \\
\text { espacios tanto de servicio social y } \\
\text { prácticas profesionales como en trabajos } \\
\text { de investigación, capacitación y que } \\
\text { brinden acompañamiento a los ejidatarios } \\
\text { a transitar hacia el autodesarrollo" }\end{array}$ & C11E1 \\
\hline
\end{tabular}

Tabla 3 Vinculación con organizaciones

\section{d) Acompañamiento docente}

El acompañamiento hace referencia a que el profesorado que labora en la institución, deben estar en constante supervisión y su función debe estar enfocada a dirigir las acciones del estudiantado para el logro de los objetivos que se han planteado, además de la vinculación con organizaciones. En otras palabras, el acompañamiento tiene que ser antes, durante y después de las intervenciones. 
Antes, guiando a los estudiantes en el proceso de sensibilización hacia las problemáticas sociales, ayudándolos en la vinculación con organizaciones y supervisando el diseño de estrategias; durante, revisando que se cumplan las actividades; y después, al realizar la evaluación y seguimiento

del impacto de los proyectos, así como la de satisfacción de los usuarios y organizaciones. (Ver tabla 4)

\begin{tabular}{|c|c|}
\hline Unidad de Análisis & Caso/Código \\
\hline $\begin{array}{l}\text { "se realiza el acompañamiento y seguimiento } \\
\text { en cada cuatrimestre" }\end{array}$ & C2E11 \\
\hline $\begin{array}{l}\text { "Seguimiento y evaluación del plan de } \\
\text { trabajo de los estudiantes" }\end{array}$ & C3E6 \\
\hline $\begin{array}{l}\text { "Los maestros que la integraban fueron los } \\
\text { responsables de planear la dinámica de } \\
\text { trabajo y se decidió continuar con equipos de } \\
\text { seis o siete alumnos". }\end{array}$ & C10E11 \\
\hline $\begin{array}{l}\text { "Los docentes sólo guían, sugieren, orientan } \\
\text { a los estudiantes, los organizan, gestionan y } \\
\text { trabajan con los alumnos en el asambleísmo, } \\
\text { reuniones con directivos de los órganos del } \\
\text { ejido, etcétera" }\end{array}$ & C11E4 \\
\hline $\begin{array}{l}\text { "Estructuración de la propuesta técnica y } \\
\text { presentación ante la dependencia } \\
\text { responsable para el dictamen y la } \\
\text { aprobación" }\end{array}$ & C11E11 \\
\hline $\begin{array}{l}\text { "se llevan a cabo las visitas de campo bajo la } \\
\text { supervisión de los docentes responsables, } \\
\text { para asegurar el buen desempeño de los } \\
\text { alumnos y el desarrollo del proyecto" }\end{array}$ & C12E15 \\
\hline
\end{tabular}

Tabla 4 Acompañamiento docente

\section{e) Diálogo social}

Para evidenciar el proceso de responsabilización social del estudiantado, es necesario la generación de espacios de diálogo para compartir las experiencias que se desarrollaron, los cuales deberán ser generados por la universidad; extendiéndose a las organizaciones y grupos vulnerables con los que se desarrollaron los proyectos de responsabilidad social. (Ver tabla 5)

\begin{tabular}{|c|c|}
\hline Unidad de Análisis & Caso/Código \\
\hline $\begin{array}{l}\text { "se realizan eventos académicos para hacer } \\
\text { balances sobre los aprendizajes y } \\
\text { posibilidades de mejora de cada proyecto } \\
\text { social de formación" }\end{array}$ & C3E13 \\
\hline $\begin{array}{l}\text { "Desarrollo de jornadas de lecciones } \\
\text { aprendidas" }\end{array}$ & C5E7 \\
\hline $\begin{array}{l}\text { "Cada equipo de estudiantes construye un } \\
\text { informe que recoge la memoria del proceso } \\
\text { vivido" }\end{array}$ & C5E16 \\
\hline $\begin{array}{l}\text { "balance del trabajo semestral con las } \\
\text { organizaciones sociales y comunitarias" }\end{array}$ & C3E7 \\
\hline $\begin{array}{l}\text { "Diálogo permanente con instituciones } \\
\text { públicas y privadas" }\end{array}$ & C5E4 \\
\hline "diálogo constante con la comunidad" & C6E2 \\
\hline
\end{tabular}

Tabla 5 Diálogo social

\section{f) Aplicación del conocimiento}

Las estrategias diseñadas deben ser ideadas con base en los conocimientos del área en la que el estudiantado se está formando dentro de las universidades, para con esto, permitirles generar espacios donde, además de ayudar a sectores vulnerables, puedan llevar a la práctica lo que aprenden en el aula. (Ver tabla 6)

\begin{tabular}{|l|l|}
\hline \multicolumn{1}{|c|}{ Unidad de Análisis } & Caso/Código \\
\hline $\begin{array}{l}\text { "los estudiantes pueden llevar a la } \\
\text { práctica los conocimientos, habilidades, }\end{array}$ & C7E14 \\
actitudes y competencias desarrolladas" & \\
\hline $\begin{array}{l}\text { "Siento una gran satisfacción de poder } \\
\text { aportar algo de mis conocimientos y al } \\
\text { mismo tiempo orientar e inculcar en los } \\
\text { alumnos a mi cargo" }\end{array}$ & \\
\hline $\begin{array}{l}\text { "Ellos se sienten muy capaces para } \\
\text { aplicar, en la vida real, los conocimientos } \\
\text { adquiridos en el aula" }\end{array}$ & C12E22 \\
\hline
\end{tabular}

Tabla 6 Aplicación del conocimiento

\section{g) Evaluación}

Para conocer el impacto que tienen las intervenciones realizadas en el campo, se necesita valorar el cumplimiento de objetivos, el trabajo en equipo y el impacto de las intervenciones. A su vez, es necesario que las organizaciones y beneficiarios evalúen el desempeño de las y los practicantes en el escenario, garantizando con ello una retroalimentación significativa, pertinente e integradora. (Ver tabla 7)

\begin{tabular}{|c|c|}
\hline Unidad de Análisis & Caso/Código \\
\hline $\begin{array}{l}\text { "evaluación que desde una perspectiva } \\
\text { multiactoral" }\end{array}$ & C5E8 \\
\hline $\begin{array}{l}\text { "Los alumnos, al terminar el periodo escolar, } \\
\text { presentan un video, integrando toda la } \\
\text { información de su trabajo y exponen su } \\
\text { aprendizaje" }\end{array}$ & C10E20 \\
\hline $\begin{array}{l}\text { "El impacto en la formación de los estudiantes } \\
\text { se refleja en el desempeño del grupo de } \\
\text { trabajo" }\end{array}$ & C12E18 \\
\hline $\begin{array}{l}\text { "La retroalimentación es una condición que } \\
\text { ahora es parte del ambiente de trabajo, donde } \\
\text { los comentarios son constructivos y se } \\
\text { observa la disposición de los actores del } \\
\text { proceso" }\end{array}$ & C12E23 \\
\hline $\begin{array}{l}\text { "Se evalúan anualmente y se desarrollan en } \\
\text { favor de los objetivos de las organizaciones } \\
\text { sociales y comunitarias" }\end{array}$ & C3E12 \\
\hline 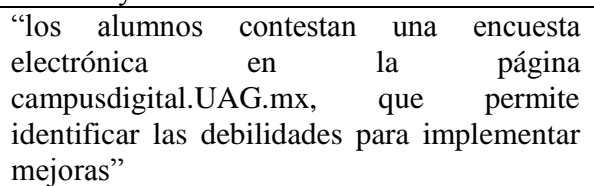 & C8E9 \\
\hline $\begin{array}{l}\text { "se les aplica una encuesta que permite } \\
\text { detectar los errores que se tienen y } \\
\text { corregirlos" }\end{array}$ & C8E10 \\
\hline
\end{tabular}

Tabla 7 Evaluación

ROJO-CARLÓN, José María, RIVERA-IRIBARREN, Maricel y CALDERÓN-SOTO, Lorena. Formación de estudiantes universitarios en responsabilidad social: Hacia la construcción de una propuesta metodológica . Revista Teoría Educativa. 2020 
Estas categorías, generadas por la intersubjetividad dentro de los proyectos de RSU analizados, brindan elementos para que el profesorado las incluyan en el diseño de procesos de formación. Para considerar estas experiencias como una guía para la práctica docente es necesario estructurarlas de tal manera que muestren un proceso de formación a seguir bajo el marco de la RS.

En la figura 1 se muestra la propuesta de estructuración de las categorías emergentes para el desarrollo de procesos de formación en RS, con acciones concretas para facilitar su desarrollo. En primer lugar, se presenta, como parte de la empatía comunitaria, el planteamiento del problema social que afecta al contexto donde se establece la universidad. En este punto se responderá a la pregunta ¿qué problemas atraviesa la sociedad actual? El papel del profesor es orientar y sensibilizar a las y los estudiantes, mostrando el posible impacto de sus acciones para mejorar las situaciones de desventaja social.

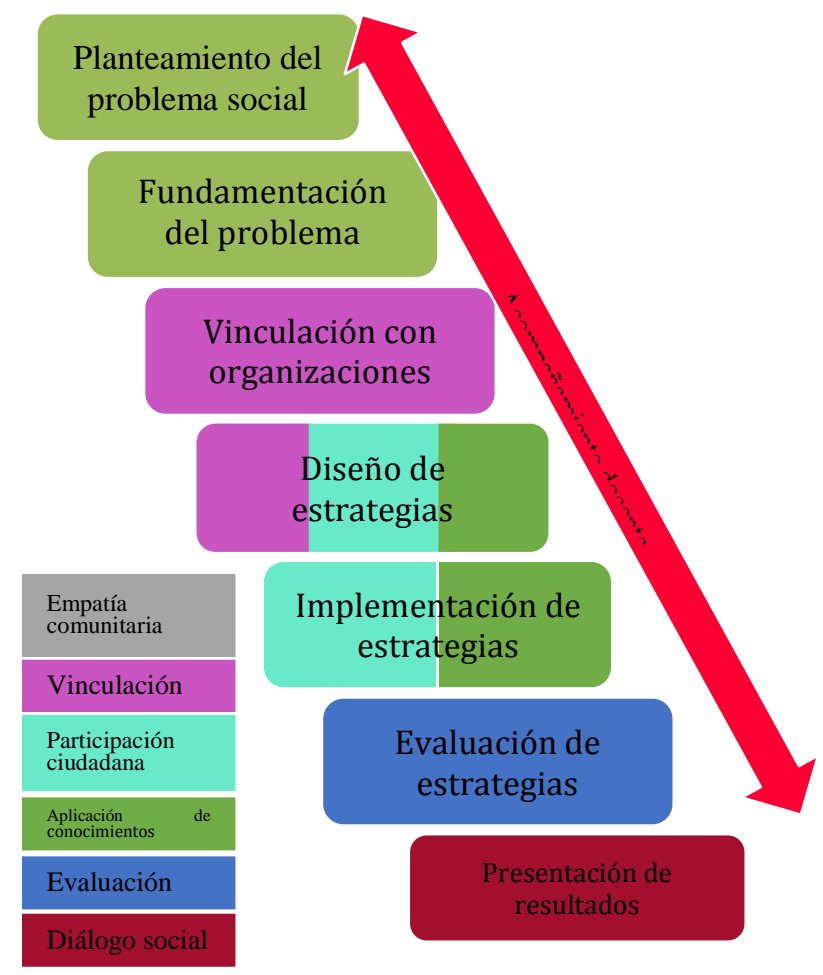

Figura 1 Propuesta de estructuración de las categorías emergentes para el desarrollo de procesos de formación en RS

El proceso inicia, desde la empatía comunitaria, planteando y fundamentando el problema social.
En esta etapa las y los estudiantes indagarán de manera documental datos empíricos sobre la problemática seleccionada, tales como estadísticas, noticias, reportes, entre otros, que los ayuden a demostrar por qué esa situación constituye un problema social, por qué es necesario solventarlo y qué se está haciendo al respecto. Una vez establecido lo anterior se procede a contactar a organizaciones civiles o dependencias gubernamentales que se muestran activas en la implementación de acciones para contribuir a solucionar el problema presentado anteriormente. Estas organizaciones brindarán orientación en los siguientes pasos con base en las experiencias que ya se hayan realizado dentro del campo al que los estudiantes apenas se introducirán.

En el cuarto elemento del proceso comienza a manifestarse la participación ciudadana para la solución de problemas. Esta etapa consiste en el diseño de estrategias para contribuir a la solución del problema social detectado. Estas estrategias deberán tomar en cuenta el campo disciplinar de los estudiantes y deberá ser validado por las organizaciones con las que se vinculó anteriormente así como por las y los profesores a su cargo.

Una vez validadas las estrategias, tanto por docentes como por miembros de las organizaciones, se procede a la implementación. En esta parte se interactúa con el grupo social que se relaciona con las problemáticas sociales trabajando de manera colaborativa en la ejecución de las propuestas diseñadas.

Una vez finalizada la etapa de implementación, se procederá a evaluar el impacto que generó, es decir, en qué medida las acciones llevadas a cabo contribuyeron a la solución de las situaciones de desventaja planteadas en la fundamentación. Siendo este el momento donde las y los profesores evaluarán el desempeño del alumnado, tomando en cuenta el impacto de cada una de las acciones realizadas no solo en la comunidad, sino durante todo el proceso de diseño.

Además de considerar la perspectiva de la organización y la satisfacción de los beneficiarios directos; esto con el fin de determinar en qué medida se cumplieron los objetivos, así como la pertinencia y efectividad de la intervención para todos los involucrados. 
Finalmente, para fomentar el diálogo social, deberán generarse espacios de conversación donde se expondrán los resultados de la implementación de estrategias. Estos foros permitirán el intercambio de experiencias y aprendizajes entre participantes, las cuales serán de utilidad en la toma de decisiones de la planta docente al momento de realizar ajustes a la planeación, esto en caso de replicar las propuestas de solución.

\section{Conclusiones}

En el estudio llevado a cabo, se lograron identificar las experiencias que destacan dentro de los procesos de enseñanza relacionados con la responsabilidad social llevados a cabo mediante proyectos comunitarios en distintas IES. Con base en el análisis de las experiencias, se generó una propuesta de orientaciones metodológicas para la formación en responsabilidad social, planteando acciones concretas que sirvan de base al profesorado en el desarrollo de intervenciones sociales.

Es importante destacar que las instituciones que buscan incluir la responsabilidad social diseñen los contenidos de las asignaturas de tal manera que el alumnado pueda desarrollar actitudes éticas y de compromiso, además, que el currículum vivido implique la solución de problemas reales relacionados al contexto (Schwalb, et. al., 2019).

Durante el proceso de formación en RS el alumnado pondrá en práctica sus competencias profesionales y mantendrá constantes interacciones con las personas con quienes colaborará de manera directa para llevar a cabo los objetivos planteados. Por ello, se recomienda poner énfasis en la ética profesional orientada al cuidado, que tome en cuenta a las personas destinatarias del servicio, como piezas que conforman la sociedad y ciudadanas del mundo.

\footnotetext{
Considerando lo planteado anteriormente, se recomienda incluir en los procesos de formación, los principios éticos definidos por López (2013) que establecen la perspectiva del individuo ante las masas, siendo estos: (1) El de beneficencia, que busca la bonanza de quienes son receptores del servicio ofrecido, tomando en cuenta los impactos que el servicio tendrá en la sociedad y en la humanidad.
}

(2) De autonomía, el cual plantea que los profesionales deberán trabajar regidos por las necesidades reales del cliente y verificando si es lo que necesita el entorno en general; y (3) De justicia, donde se pretende dar lo que le corresponde a las personas desde una perspectiva individual, social y global.

De la misma manera, se recomienda que la formación en responsabilidad social no solamente sea el compromiso de una sola asignatura o las que competen a la formación de valores, sino que debería trabajarse desde un enfoque holístico donde todas las asignaturas se complementan y aporten para el desarrollo de ésta en las y los estudiantes (Vallaeys, 2018).

Además, se sugiere al profesorado el no perder de vista que el objetivo de la formación universitaria en responsabilidad social es que las y los estudiantes puedan desarrollar por medio de acciones su empatía e interés en el cuidado del contexto en el que se desenvuelven, aplicando los conocimientos adquiridos dentro del grado que estén cursando. Por lo cual no solo es necesario incrementar las actividades en escenarios reales sino que es menester buscar motivar a los jóvenes promoviendo en ellos un compromiso social transformador, nada fácil de lograr pero no imposible, es necesario formar ciudadanos con conciencia, incentivarlos a cambiar de una motivación asistencial a una motivación transformadora, que les permita involucrarse más allá de la realización de tareas, logrando en ellos un cambio significativo en la forma de ver la problemática social, que les permita involucrarse, reflexionar y actuar sobre el impacto que su práctica profesional tendrá en un futuro inmediato (Apodaka, 2020).

Como reflexión final, es importante destacar el papel de la formación previa a la universitaria, el pérfil de un profesional socialmente responsable no puede gestarse con solo cuatro años de preparación, si bien se pueden obtener resultados satisfactorios, también es cierto que se debe construir sobre bases sólidas. El mundo se encuentra ante circunstancias nunca antes vistas, la pandemia del 2020 hace reflexionar sobre los alcances de la humanidad, la educación no puede ser la excepción, hoy más que nunca se debe tener conciencia de que se prepara a seres humanos para un mundo que no existe, en el que día a día se generan cambios que afectan de manera global. 
De ahí la importancia de formar desde edad temprana, sentar las bases de la empatía, la solidaridad, el respeto y la identidad comunitaria, serán esenciales para los profesionistas del mañana, no basta llenarlos de conocimientos, ni habilidades cognitivas, es necesario enfocarse en el aprender a ser, pero no un ser aislado sino un ser social capaz de adaptarse a las nuevas realidades y no aislarse en un mundo que lo invita a realizar todo desde casa.

Este trabajo representa un avance para el establecimiento de un modelo de formación en responsabilidad social, planteando las categorías que se deberían tomar en cuenta para desarrollar toda acción social, dirigida desde la universidad hacia la comunidad a través de la práctica educativa: empatía comunitaria, participación ciudadana, vinculación con organizaciones, acompañamiento docente, diálogo social y aplicación del conocimiento.

\section{Referencias}

Ahumada, E., Ravina, R. y López, M. (2018). Responsabilidad Social Universitaria. Desarrollo de competitividad organizacional desde el proceso educativo. Revista Electrónica Actualidades Investigativas en Educación, 18 (3).

Alférez, A. (2014). La participación social curricular en la Responsabilidad Social Universitaria: El caso de la Facultad de Educación de la Universitat Internacional de Catalunya (Tesis doctoral). Universitat Internacional de Catalunya, Barcelona.

Apodaka, M. (2020). El voluntariado en el ámbito educativo material dirigido al profesorado par. Fevocam.org. Obtenido el 11 de octubre de 2020, de https://www.fevocam.org/download/103/materi al-didactico/3154/el-voluntariado-en-el-ambitoeducativo-material-dirigido-al-profesorado.pdf.

Asociación Nacional de Universidades e Instituciones de Educación Superior. (2016). Prácticas y modelos de Responsabilidad Social Universitaria en México. OMERSU.

Ayala-Rodríguez, N., Barreto, I., Rozas, G., Castro, A. y Moreno, S. (2019). Social transcultural representations about the concept of university social responsibility. Studies in higher education, 44(2).
Barroso, F. G., Santos, R. A. y Patrón, R. M. (2016). Fortalecimiento de la Responsabilidad Social Universitaria. Un estudio en el Sureste de México. Revista RAITES, 2(4).

Beltrán, J., Iñigo, E. y Mata, A. (2014). La responsabilidad social universitaria, el reto de su construcción permanente. Universia, 5(14), pp. $3-18$.

Calles, F., Ortiz, A., y Félix, S. (2018). Responsabilidad Social Universitaria en el currículo la Licenciatura en Contaduría Pública. Caso del Departamento de contabilidad de la Universidad de Sonora. México. Asociación de Profesores de Contaduría y Administración de México (APCAM).

Gaete, R. (2017). Responsabilidad social universitaria: una nueva mirada a la relación de la universidad con la sociedad desde la perspectiva de las partes interesadas. Un estudio de caso (Tesis doctoral). Universidad de Valladolid, España.

Gaete, R. (2015). La responsabilidad social universitaria desde la perspectiva de las partes interesadas: un estudio de caso. Costa Rica. Universidad de Costa Rica.

Guzmán Droguett, María Angélica, Maureira Cabrera, Óscar, Sánchez Guzmán, Alejandra, \& Vergara González, Adriana. (2015). Innovación curricular en la educación superior: ¿Cómo se gestionan las políticas de innovación en los (re)diseños de las carreras de pregrado en Chile?. Perfiles educativos, 37(149), 60-73. Recuperado en 11 de noviembre de 2020, de http://www.scielo.org.mx/scielo.php?script=sci _arttext\&pid=S0185-

26982015000300004\&lng=es\&tlng=es.

Hernández, R., Fernández, C. y Baptista, M. P. (2014). Metodología de la investigación (6ta ed.). McGraw-Hill.

López, M. (2013). Ética profesional y complejidad, los principios y la religación. Perfiles educativos, 35(142), 43-52.

Manig, A., Márquez, L., y Madueño, M. L. (2018). Métodos de investigación cualitativa: la comprensión de las voces de los actores en ambientes educativos. México: Pearson.

ROJO-CARLÓN, José María, RIVERA-IRIBARREN, Maricel y CALDERÓN-SOTO, Lorena. Formación de estudiantes universitarios en responsabilidad social: Hacia la construcción de una propuesta metodológica . Revista Teoría Educativa. 2020 
Ojeda, J. y Álvarez, D. (2015). Responsabilidad social en las universidades: antecedentes, trayectorias y perspectivas. México. Revista de la Comisión Estatal para la Planeación de la Educación Superior del Estado de Guanajuato (COEPES).

Pérez, J. (2016). Responsabilidad Social universitaria. México: Universidad Autónoma de Yucatán (UADY).

Schwalb, M. M., Prialé, M. A. y Vallaeys, F. (2019). Guía de Responsabilidad Social Universitaria (1.era Ed). Perú: Universidad del Pacífico.

Sosik, J. J., Koul, R. y Cameron, J. C. (2017). Gender and Contextual Differences in Social Responsibility in Thai Schools: A Multi-Study Person versus Situation Analysis. Journal of Beliefs y Values, 38(1).

Vallaeys, F. (2014). La responsabilidad social universitaria: un nuevo modelo universitario contra la mercantilización. Revista Iberoamericana de Educación Superior, V (12).

Vallaeys, F. (2018). Las diez falacias de la Responsabilidad Social Universitaria. Revista Digital de Investigación en Docencia Universitaria, $12(1), \quad 34-58 . \quad$ DOI: http://dx.doi.org/10.19083/ridu.12.716.

Vallaeys, F. y Álvarez Rodríguez, J. (2019). Hacia una definición latinoamericana de responsabilidad social universitaria. Aproximación a las preferencias conceptuales de los universitarios. Educación XX1, 22(1), pp. 93-116, DOI: 10.5944/educXX1.19442

\section{Agradecimiento}

Este proyecto fue financiado por el Programa para el Fomento y Apoyo a Proyectos de Investigación del Instituto Tecnológico de Sonora (PROFAPI 2020). 